\title{
Entwicklung von Versuchsständen zur natürlichen und künstlichen Bewitterung umlaufender Faserseile
}

\author{
Uwe Metzner* \\ Sächsisches Textilforschungsinstitut e.V., Chemnitz, Deutschland \\ * Korrespondenz: uwe.metzner@stfi.de
}

Erhalten am 13. Oktober 2020; Angenommen am 14. Oktober 2020; Online zugänglich seit 7. Dezember 2020

(C) 2020 by U. Metzner. Dies ist ein Open-Access-Artikel, der unter der Creative Commons Attribution License (CC-BY 4.0) verbreitet wird, die die uneingeschränkte Nutzung, Verbreitung und Vervielfältigung in jedem Medium erlaubt, vorausgesetzt, das Originalwerk wird ordnungsgemäß zitiert.

\begin{abstract}
Die Bewitterungsprüfung von Faserseilen hat besondere Relevanz, da derzeit noch keine allumfassenden Erkenntnisse vorliegen, welchen Einfluss UV-Belastung, Temperatur und Feuchte beziehungsweise Regen auf die Lebensdauer von Seilen aus Hochleistungsfasern in Freiluftanwendungen oder in Betriebsbereichen mit erhöhtem Aufkommen von UV-Strahlung beziehungsweise Tageslicht haben. Stand der Technik vor dem Forschungsvorhaben war die Bewitterung von statischen Seilproben. Diese wurden fest eingespannt und während der Bewitterungsdauer nicht bewegt, wodurch diese Bewitterungsversuche die realen Verhältnisse bei der Anwendung von Faserseilen jedoch nicht hinreichend abbilden. So erfolgt bei einer starren Probeneinspannung die Einwirkung von Sonnenstrahlen nur einseitig. Darüber hinaus konnte durch die herkömmlichen Bewitterungsverfahren die Schutzwirkung des Seilmantels unter Gebrauchsbedingungen ebenfalls nicht hinreichend realitätsnah geprüft werden. Der Artikel hat die Problemstellung und die konstruktive Entwicklung der Gerätetechnik für die natürliche sowie die labortechnische Bewitterung umlaufender Faserseile zum Gegenstand und geht abschließend auf die Ergebnisse erster Bewitterungsversuche ein. Die Technologie sowie die Versuchsgerätetechnik zur künstlichen Bewitterung von Faserseilen stehen nun ersten Anwendung zur Verfügung. Bei Bedarf besteht die Möglichkeit zur Erhebung von Referenzparametern durch natürliche Bewitterung.
\end{abstract}

KEYWORDS Faserseile, Laborbewitterung, natürliche Bewitterung, Ablegereife

\section{Einleitung}

Ziel des Forschungsprojektes war die Entwicklung einer neuartigen Technologie und zugehöriger Gerätetechnik zur Bewertung der Eigenschaften von Faserseilen und deren Degradation unter dem Einfluss künstlicher und natürlicher Bewitterung.

Am Markt ist nach wie vor ein enormer Anstieg des Bedarfs an Faserseilen für technische Anwendungen $\mathrm{zu}$ erkennen. Waren Faserseile vor einigen Jahren maßgeblich für marine Anwendungen oder für den Einsatz im Sport- und Freizeitbereich nachgefragt, gehen die Entwicklungen zukünftig in technische Applikationen. Neben den textilphysikalischen Eigenschaften, wie z.B. Kraft- Dehnungsverhalten, sind der Nachweis von Gebrauchseigenschaften unter Umweltbelastung Voraussetzung für die Erschließung dieser neuen Märkte 
im Outdoorbereich. Das STFI e.V. hat bereits im Rahmen des INNO-Zug-Projektes des Lehrstuhls für Förder- und Materialflusstechnik an der Technischen Universität Chemnitz mit der Entwicklung neuartiger Faserseile und der Bewertung deren Eigenschaften mitgewirkt. Die Bewitterungsprüfung von Faserseilen hat besondere Relevanz, da derzeit keinerlei Erkenntnisse vorliegen, welchen Einfluss UV-Belastung, Temperatur und Feuchte bzw. Regen auf die Lebensdauer von Faserseilen in Freiluftanwendungen oder in Betriebsbereichen mit erhöhtem Aufkommen von UV-Strahlung bzw. Tageslicht haben.

Die Einflüsse mechanischer Beanspruchungen, wie zum Beispiel Biege- und zyklische oder schlagartige Zugbelastungen, auf die Lebensdauer von Faserseilen werden im Rahmen von Forschungsprojekten an der Technischen Universität Chemnitz und am STFI e.V. untersucht. Ebenfalls wurden erste Untersuchungen zur Auswirkung von Witterungseinflüssen auf die Festigkeit von Faserseilen aus hochfesten Fasern (Dyneema ${ }^{\circledR}$, Vectran $^{\mathrm{TM}}$, Technora) durchgeführt. Dabei hat sich gezeigt, dass schon die Bewitterung von Seilen, die nicht unter mechanischer Last stehen, einen erheblichen Einfluss auf die Festigkeit der Seile haben kann. Weiterhin wurde festgestellt, dass sich extreme Temperaturen $\left(+70^{\circ} \mathrm{C},-30^{\circ} \mathrm{C}\right)$ ohne die Einwirkung von UV-Strahlung und/oder Wasser negativ auf die Festigkeit von Seilen auswirken. Darüber hinaus ist bei einigen Werkstoffen, zum Beispiel bei Polyamiden, bekannt, dass diese bei Kontakt mit Wasser bis zu $40 \%$ ihrer Festigkeit verlieren.

Es ist nun davon auszugehen, dass sich der schädigende Einfluss von Licht auf die polymeren Werkstoffe mit denen durch die Belastung und den daraus resultierenden Verschleiß- und Schädigungsmechanismen überlagert und zusätzlich einen negativen Einfluss auf die Festigkeit hat.

Bei der weiterführenden Entwicklung der Faserseile ist zu untersuchen, ob und inwieweit die durch die Seilbelastung auftretenden Mechanismen, wie zum Beispiel Verschleiß, durch die Witterungseinflüsse noch verstärkt werden. Dafür war die Entwicklung einer Technologie zur gezielten Bewitterung von bewegten Faserseilen erforderlich, da bisher am Markt keine entsprechenden Prüfsysteme verfügbar waren, welche die Prüfung bei gleichzeitiger Bewegung ermöglichen. Die Entwicklung einer solchen neuartigen Bewitterungs - und Prüftechnologie schloss notwendigerweise die konstruktive Entwicklung sowie die Realisierung von entsprechenden labortechnischen Prüfsystemen und Versuchsanlagen mit ein.

Die Entwicklung von Prüfstand und Verfahren ermöglicht nun die Verifizierung der künstlichen Bewitterung im Vergleich zur Freiluftbewitterung und bildet damit die realen Anwendungsbedingen $\mathrm{ab}$.

Anwendungen, bei denen zur Dimensionierung und Abschätzung der Lebensdauer sowie zum Nachweis von sicherheitsrelevanten Eigenschaften ein solcher Prüfstand notwendig ist, sind vor allem Seile, welche in technischen Einrichtungen vorwiegend auf Außengeländen zum Einsatz kommen. So zum Beispiel auf Krananlagen für Baustellen, Güterbahnhöfen und Hafenanlagen. Weitere Anwendungsgebiete der neuartigen Faserseile liegen in den Bereichen des außenliegenden Sonnenschutzes. Darüber hinaus sind Faserseile auch auf dem Vormarsch für Winden in forsttechnischen Anwendungen, der Schlepp- und Bergetechnik, sowie im Kfz-Bereich mit der Anforderung einer hohen Verfügbarkeit. 
Für all diese Einsatzgebiete ist es zwingend erforderlich, die jeweils relevanten technischen Eigenschaften der Faserseile unter sogenannten Gebrauchsbedingungen angeben zu können. Zur Gewährleistung der technischen Sicherheit ist es erforderlich den kombinierten Einfluss von Licht, Feuchte/Regen und Temperatur auf die jeweiligen Materialien zuverlässig ermitteln zu können.

Ziel der Entwicklung der neuartigen Prüftechnik und Technologie zur künstlichen Bewitterung von bewegten Faserseilen war es somit auch, die benötigte Prüfdauer gegenüber einer Freibewitterung signifikant zu reduzieren, um die Entwicklungszeiten für Faserseile maßgeblich zu verkürzen und eine höhere Wirtschaftlichkeit zu erreichen.

\section{Konstruktive Entwicklung und Realisierung eines Außenprüfstandes für die Langzeitbewitterung bewegter Faserseilproben}

Zunächst war die erforderliche Prüflänge der Seilproben für statistisch gesicherte Untersuchungen der mechanischen Eigenschaften der Seile vor und nach der Bewitterung festzulegen. Ausgehend von der erforderlichen Probenlänge für die jeweiligen mechanischen Prüfungen, wie beispielsweise dem Zugversuch, sowie der jeweils notwendigen Probenanzahl, wurde festgelegt, dass die Gesamtprobenlänge für die Freiluftbewitterungsversuche 100 Meter betragen soll.

Bei einer einfachen, mäanderförmigen Anordnung der Faserseile hätte dies eine Fläche von ca. 30 Quadratmetern erfordert. Dabei war zu berücksichtigen, dass dann auch der Antrieb der einzelnen, auf dieser Fläche verteilt angeordneten, Seilumlenkrollen sehr großräumig hätte ausgelegt werden müssen.

Durch die Anwendung von Seilumlenkrollen mit jeweils drei unterschiedlichen Durchmessern, konnte eine Lösung erarbeitet werden, welche die Aufnahme der dreifachen Seillänge pro Fläche ermöglicht. Die erforderliche Gesamtfläche konnte somit auf ca. 10 Quadratmeter reduziert werden. Um die Montage sowie die Handhabung des Versuchsstandes und der jeweiligen Seilproben weiter zu vereinfachen, wurde der Versuchsstand als modulares System konzipiert und konstruktiv entwickelt, wobei ein Modul jeweils drei

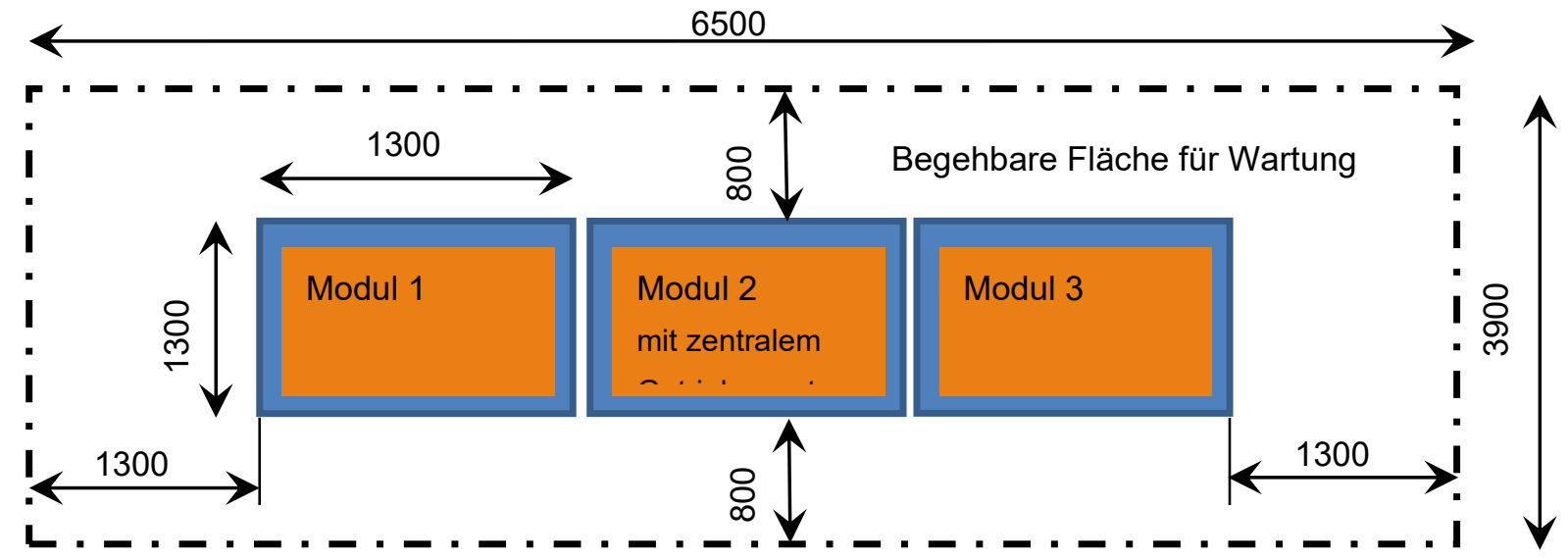

Abbildung 1: Layout für die Aufstellung des Außenprüfstandes im Gelände 


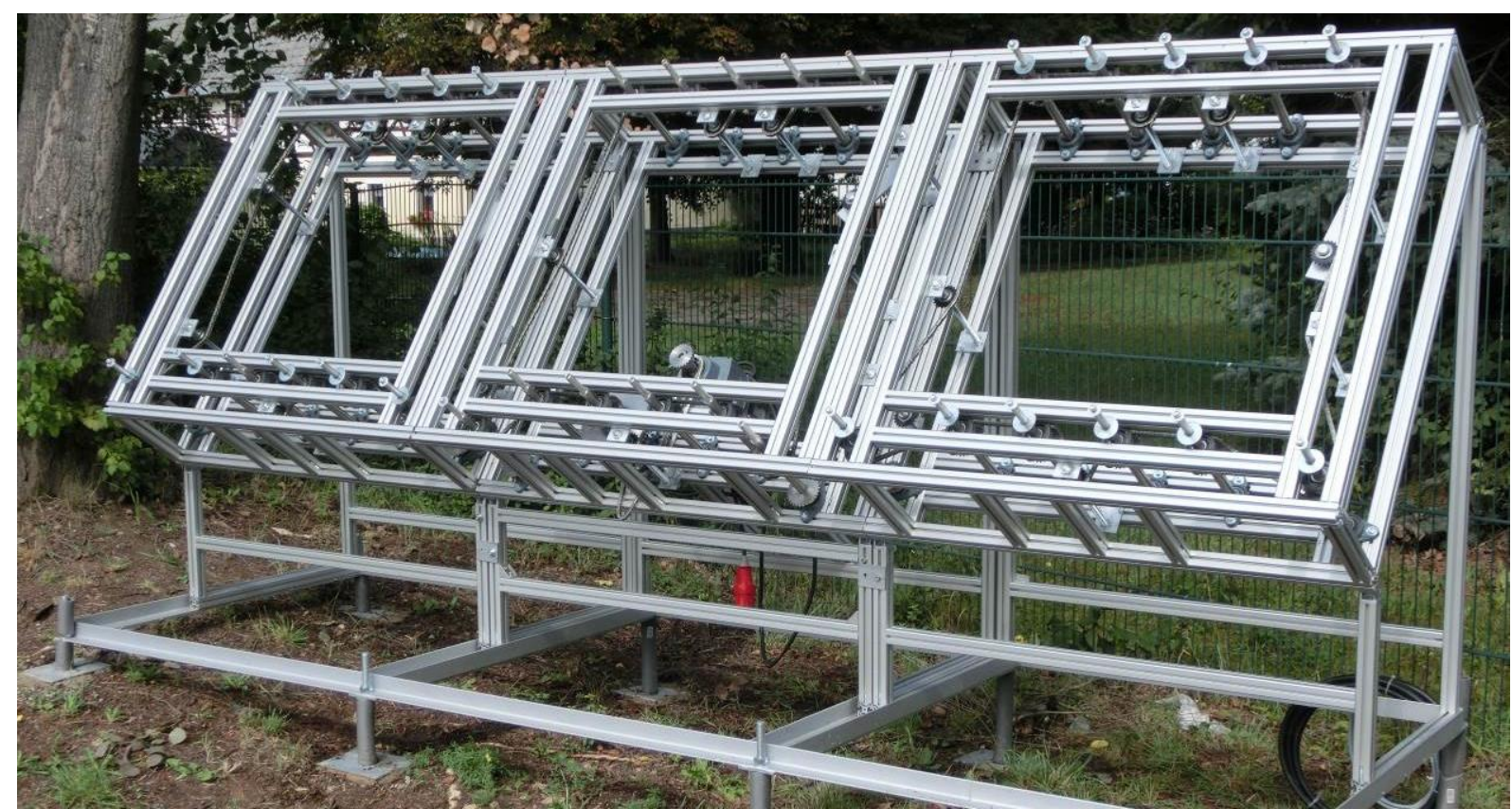

Abbildung 2: Aufstellung des Außenprüfstandes im Gelände des STFI

Faserseile mit einer Gesamtlänge von über 34 Metern Länge aufnehmen kann. Um eventuell auftretende Schlupfeffekte zwischen den umlaufenden Seilproben und den jeweiligen Seilscheiben zu vermeiden, wurde der Versuchsstand so entwickelt, dass alle Seilscheiben durch eine umlaufende Antriebskette angetrieben werden.

Nach der konstruktiven Entwicklung des Außenprüfstandes im CAD-System wurde dessen Aufstellungslayout im Gelände geplant. Ursprünglich war vorgesehen, jedes der drei Module mit einem eigenen Antriebsmotor auszurüsten. Da die Kosten für je eine Antriebseinheit jedoch weit höher ausfielen, als ursprünglich geplant, wurde ein zentraler Antrieb für alle drei Module realisiert. Die nachfolgende Abbildung 1 enthält den Entwurf für die Aufstellung des Außenprüfstandes im Gelände.

Für die Aufstellung der drei Module des Außenprüfstandes wurde ein gemeinsames Untergestell konstruiert und in der Werkstatt des STFI hergestellt. Danach erfolgte die Montage des Stahluntergestells, dessen Justierung sowie die Montage der drei Module des Außenprüfstandes. Siehe auch die Abbildung 2.

\section{Umrüstung eines vorhandenen labortechnischen Prüfsystems für Bewitterungsversuche an bewegten Faserseilen:}

Im Rahmen dieses Arbeitsschwerpunktes erfolgte die konstruktive Entwicklung sowie die Realisierung einer Aufnahmevorrichtung für umlaufende Faserseilproben zur Integration in die im STFI vorhandenen Prüfgeräte UV200SB (Global-UV-Testgerät) und WK3-600/70 der Firma Weiss Umwelttechnik GmbH. Zunächst war es erforderlich die optimale Anordnung der Seilscheiben zu ermitteln, welche die Aufnahme von Seilproben mit einer jeweiligen Mindestlänge von 10 Metern erlaubt. Der Antrieb der Seilscheibeneinheit erfolgt über einen Elektromotor, welcher sich außerhalb des jeweiligen Prüfgerätes befindet. Das 
Drehmoment wird über eine Welle übertragen, die den Elektromotor mit einer Kegelradstufe an der Aufnahmevorrichtung für umlaufende Faserseilproben (Seilscheibeneinheit) verbindet. Die Übertragung des Drehmomentes zu jeder Seilscheibe erfolgt durch eine Antriebskette. Die konstruktive Entwicklung der Aufnahmevorrichtung wurde mit Hilfe eines CAD-Systems vorgenommen. Siehe dazu die Abbildung 3.

Für die Durchführung der Antriebswelle vom Elektromotor zur Seilscheibeneinheit durch die Gehäuseseitenwand des jeweiligen Prüfgerätes hindurch, konnten kreisförmige Montageöffnungen genutzt werden, welche an den Geräten bereits vorhanden waren. Nach der konstruktiven Entwicklung der Aufnahmevorrichtung erfolgte deren Realisierung sowie die elektrotechnische Einbindung des Antriebsmotors in die elektrische Anlage des Bewitterungsprüfgerätes. Die Aufnahmevorrichtung für die umlaufenden Faserseilproben wird für die jeweilige Zeitdauer der Seilbewitterung in das Innere des Global-UV-Testgerätes eingesetzt. Nach dem Abschluss dieser Arbeiten wurde das umgerüstete Prüfgerät in Betrieb genommen und die entsprechenden Funktionen getestet. Siehe dazu die Abbildungen 4 bis 5 .

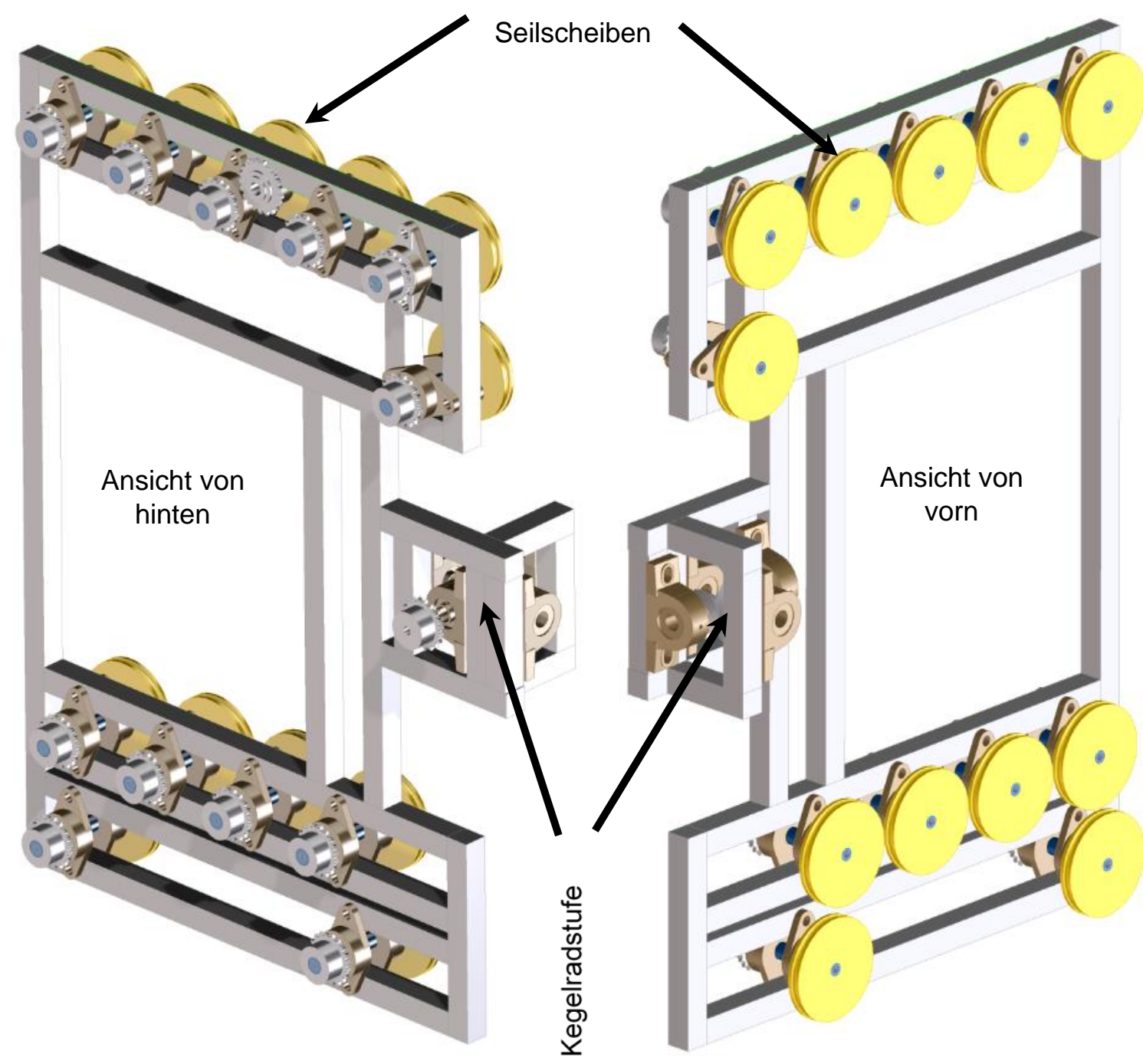

Abbildung 3: Aufnahme für umlaufende Seilproben in einem Laborgerät zur künstlichen Bewitterung 


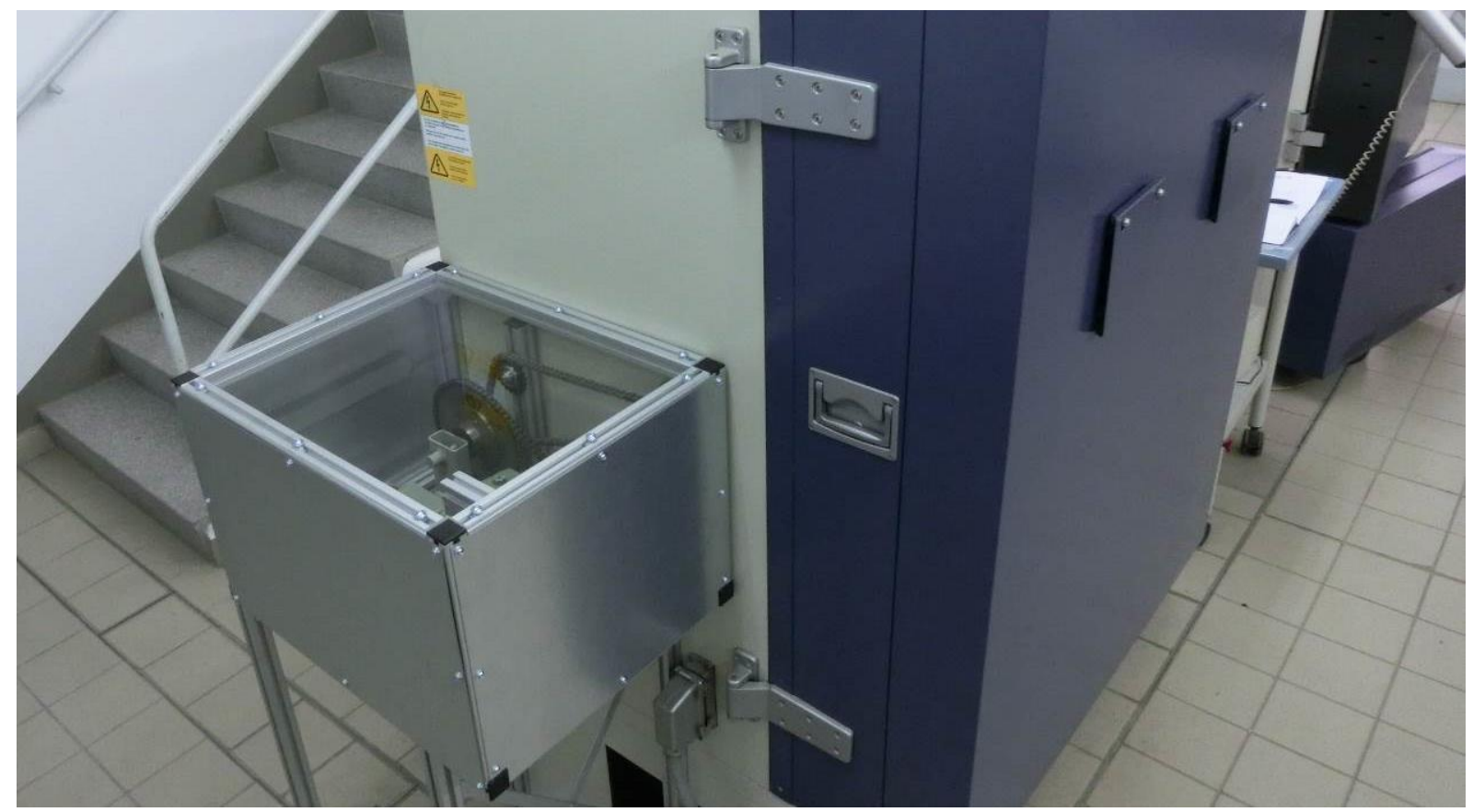

Abbildung 4: Außenliegender Antrieb für die Aufnahmevorrichtung für umlaufende Faserseilproben

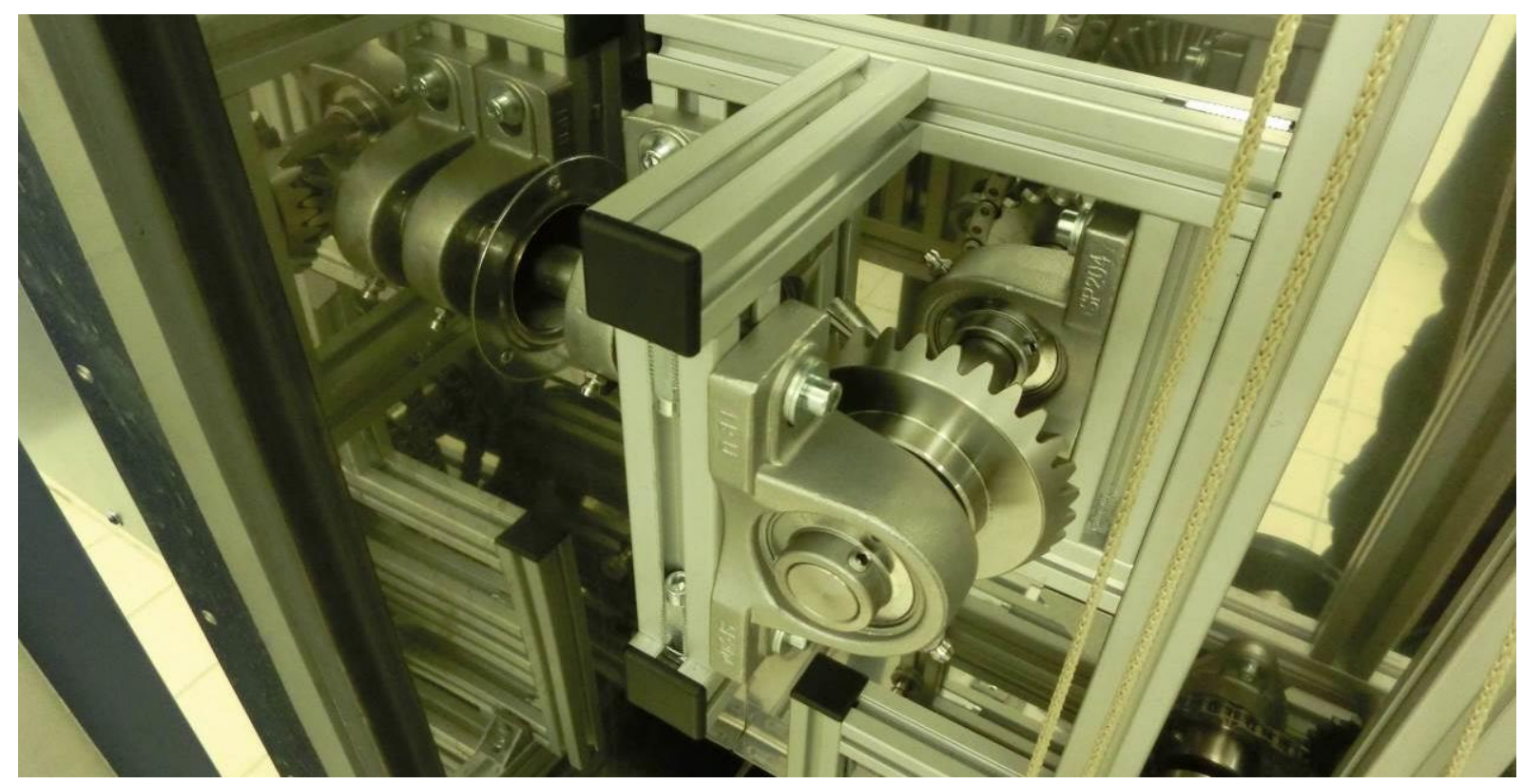

Abbildung 5: Drehmomentübertragung mittels Kegelradstufe

\section{Durchführung von Bewitterungsversuchen}

\section{Außenbewitterung umlaufender und nicht-umlaufender (starrer) Faserseilproben}

Nach der Herstellung der benötigten Prüfstände und der entsprechenden Vorbereitung der jeweiligen Seilproben konnte mit den Bewitterungsversuchen begonnen werden. Die Herstellung der benötigten Faserseilproben erfolgte an der Technischen Universität Chemnitz. Zur Bewitterung wurden die Proben auf die Prüfstände gebracht und der Elektromotor zum Antrieb der Seilscheiben gestartet. Die Bewitterungsdauer wurde auf fünf 
Monate festgelegt, um die Ergebnisse noch während der Projektlaufzeit auswerten zu können. Mehrmals wöchentlich erfolgte eine Kontrolle des Versuchsaufbaues hinsichtlich Funktionalität und zur Beseitigung von Verunreinigungen, wie Beispielsweise anhaftender Blätter. Siehe dazu die Abbildung 6.

Um die Versuchsergebnisse entsprechend vergleichen zu können, erfolgte Parallel zur Bewitterung umlaufender Faserseilproben ebenfalls die Bewitterung von nichtumlaufenden, also starren, Faserseilproben. Dafür wurden entsprechende Faserseilproben der gleichen Herstellungscharge auf Bewitterungsgestellen befestigt. Die Bewitterungsdauer für die nichtumlaufenden Faserseilproben betrug ebenfalls fünf Monate.

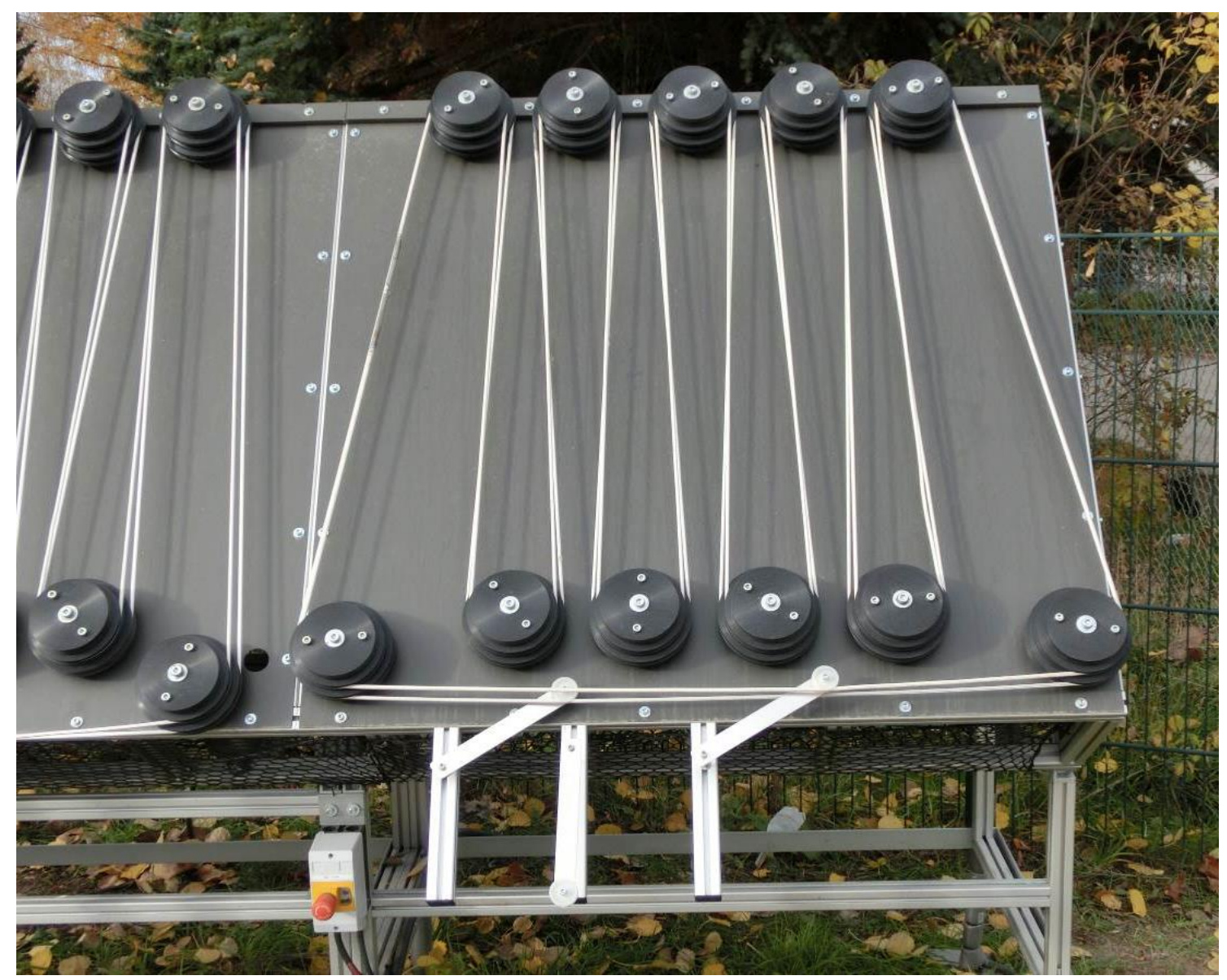

Abbildung 6: Durchführung der Bewitterung umlaufender Seilproben im Außenbereich

\section{Laborbewitterung umlaufender Faserseilproben}

Unter Einbeziehung der erzielten Ergebnisse bei der Außenbewitterung umlaufender Faserseilproben sowie der durchgeführten Vorversuche zur künstlichen Bewitterung, wurden die entsprechend zu berücksichtigenden Verfahrensparameter für die Durchführung der zweiten Versuchsreihe zur künstlichen Bewitterung umlaufender Seilproben zusammengestellt und quantifiziert. Dabei wurde die Versuchsdauer der künstlichen Bewitterung auf 20 Tage festgelegt. Dies entspricht einer Bewitterungsdauer im Freien von fünf Monaten unter Berücksichtigung der Jahreszeiten, zu denen die Freibewitterung durchgeführt wurde, siehe dazu die Tabelle 1 sowie die Abbildung 7. 
Tabelle 1: Parameter für die Bewitterung von Faserseilen im Laborbewitterungsgerät (Äquivalent einer natürlichen Bewitterung für die Dauer von fünf Monaten)

\begin{tabular}{ll}
\hline Parameter & Wert \\
\hline Bewitterungsdauer & 20 Tage \\
Bestrahlungsstärke & $45 \mathrm{~W} / \mathrm{m}^{2}$ \\
Wellenlängenbereich & $300-400 \mathrm{~nm}$ \\
Beregnungszyklen & $4 \times 60 \mathrm{~min} / \mathrm{Tag}$ \\
Temperatur - bei Beregnung & $25^{\circ} \mathrm{C}$ \\
Temperatur - ohne Beregnung & $50^{\circ} \mathrm{C} \mathrm{Schwarz-Standardtemperatur}$ \\
Geschwindigkeit der Umlaufbewegung der Faserseilproben & $1 \mathrm{~m} / \mathrm{min}$ \\
\hline
\end{tabular}

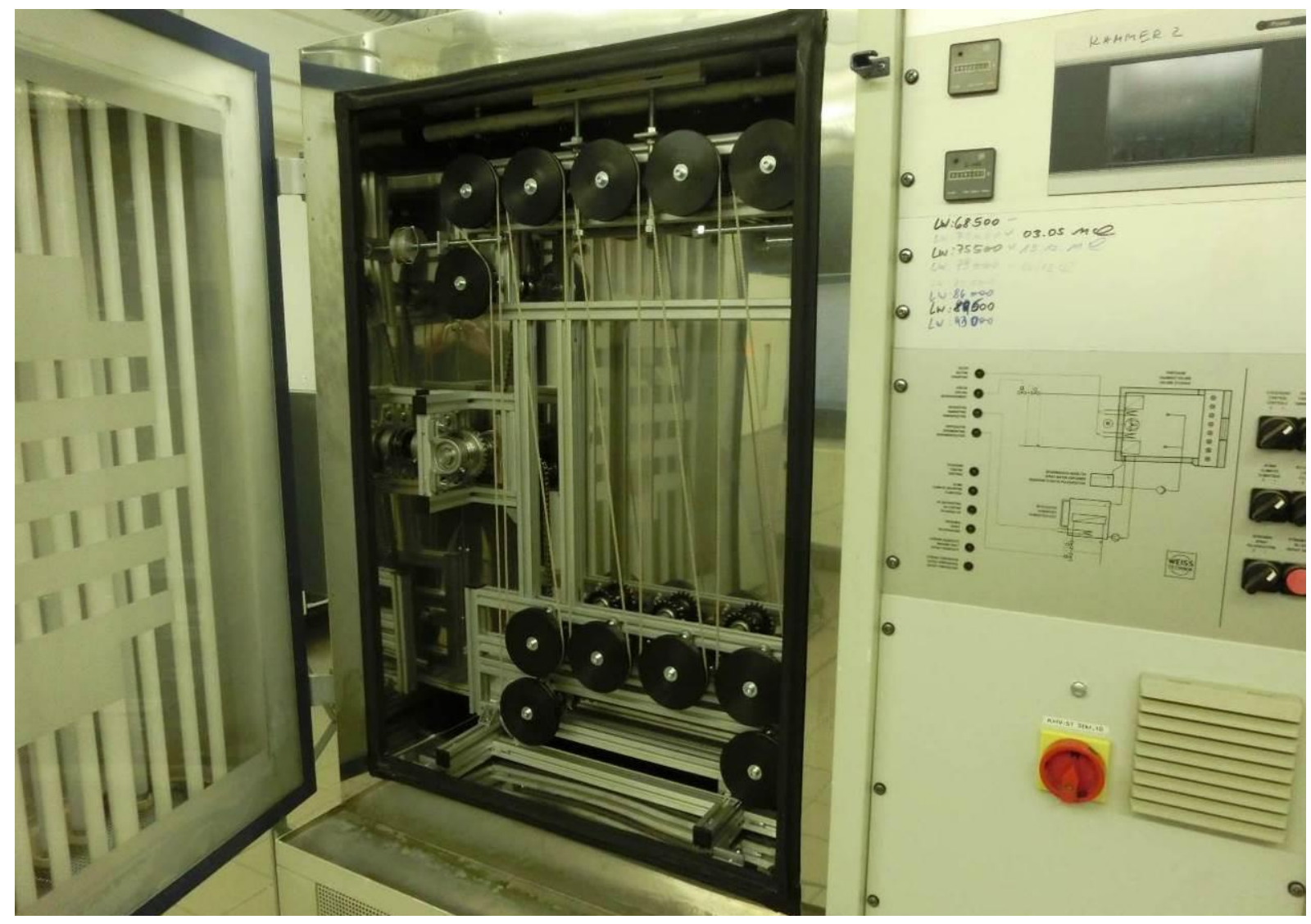

Abbildung 7: Durchführung der Laborbewitterung umlaufender Seilproben

\section{Ergebnisvergleich der Bewitterungsveruche an den jeweiligen Faserseilproben durch Zugversuche nach DIN EN ISO 2307}

Nach der Abstimmung der technischen Parameter sowie der Durchführung von Bewitterungsversuchen zur Verifizierung, wurden die jeweiligen Seilproben nach DIN EN ISO 2307 geprüft. Das entscheidende Kriterium dabei war die maximal ertragbare Zugkraft. Die Abbildung 8 zeigt beispielhaft, das Kraft-Dehnungs-Diagramm von Faserseilproben, bestehend aus einem Technora ${ }^{\circledR}$-Kern und einem PES-Mantel. 
Die Ergebnisse der Zugprüfung von Faserseilen, die aus einem Dyneema ${ }^{\circledR}$-Kern und einem Polyestermantel (PES-Mantel) bestehen, sind in Tabelle 2 zusammengefasst. Für die Seilproben mit einem Durchmesser von 3 Millimetern konnten die Zugversuche unter Einbeziehung des gesamten Probenquerschnittes durchgeführt werden. Bei den Seilproben mit einem Durchmesser von 6 Millimetern kam es jedoch, trotz entsprechender Umschlingung, zum Durchrutschen an den Seilklemmen der Zugprüfeinrichtung, weshalb die Zugprüfung auf jeweils nur eine der insgesamt zwölf Dyneema ${ }^{\circledR}$-Kernlitzen beschränkt wurde. Die Prüfergebnisse waren jedoch hinsichtlich der vergleichenden Ergebnisauswertung hinreichend repräsentativ.

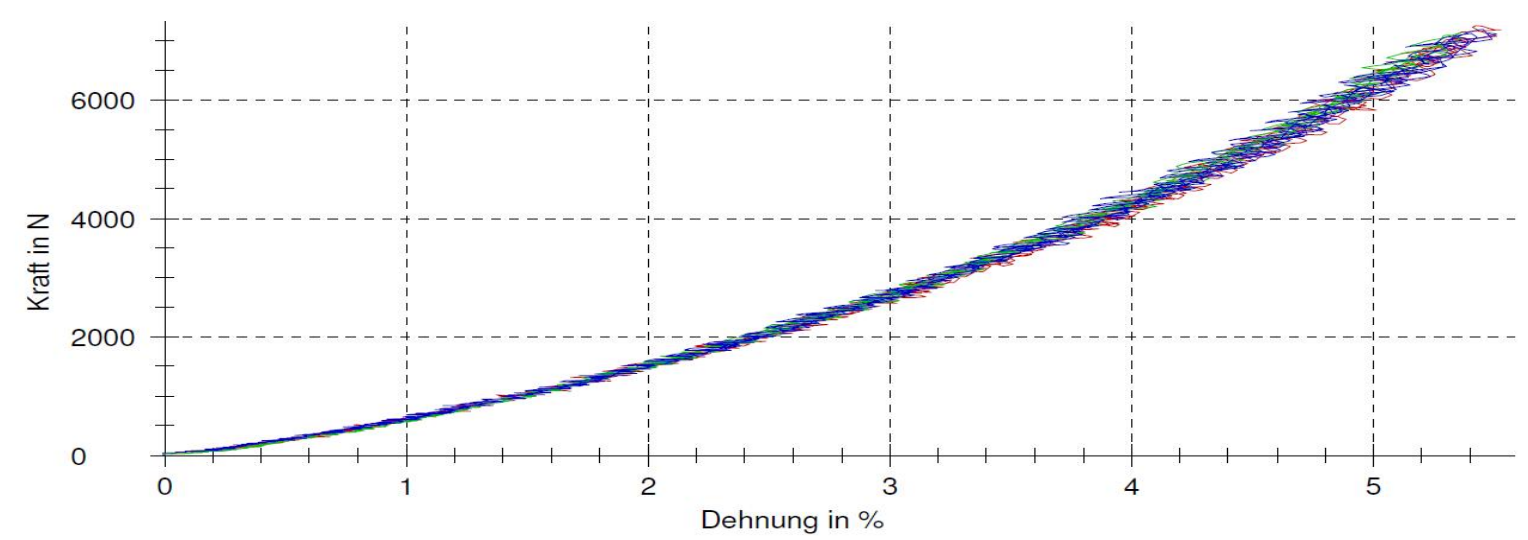

Abbildung 8: Kraft-Dehnungs-Diagramm von Proben aus einem Technora ${ }^{\circledR}$-Kern und einem PES-Mantel

Tabelle 2: Mittlere ertragbare Zugkraft von Seilproben vor und nach Bewitterungsversuchen

\begin{tabular}{lll}
\hline Probenbenennung & Bewitterungsart & Zugkraft (Mittelwert) in kN \\
\hline Dyneema ${ }^{\circledR}$-Kern / PES-Mantel / Ø $3 \mathrm{~mm}$ & unbewittert & 8,22 \\
& außen-statisch & 7,80 \\
& außen-umlaufend & 6,98 \\
& künstlich-umlaufend & 6,90 \\
\hline Dyneema ${ }^{\circledR}$-Kern / PES-Mantel / Ø6 mm & unbewittert & $3,10 \quad$ (37,2 auf 12 Kernlitzen gerechnet) \\
(1 von 12 Kernlitzen geprüft) & außen-statisch & $3,05 \quad$ (36,6 auf 12 Kernlitzen gerechnet) \\
& außen-umlaufend & $2,11 \quad$ (25,3 auf 12 Kernlitzen gerechnet) \\
& künstlich-umlaufend & $2,05 \quad$ (24,6 auf 12 Kernlitzen gerechnet) \\
\hline
\end{tabular}

\section{Zusammenfassung}

Die Auswertung der Zugversuche, welche zur Verifizierung der technischen Parameter der künstlichen Bewitterung durchgeführt wurden, hat gezeigt, dass die ertragbare Zugkraft durch die Bewitterungseinwirkung signifikant abnimmt. Die Prognose, dass die ertragbare Zugkraft nach der Bewitterung von umlaufenden Seilproben geringer ausfällt, als bei statisch bewitterten Seilproben, konnte ebenfalls bestätigt werden. Dies kann darauf zurückgeführt werden, dass die geflochtene Mantelstruktur der Seile bei umlaufenden Proben infolge der mechanischen Beanspruchung, für die jeweils einwirkenden 
Bewitterungseinflüsse durchlässiger ist, als bei starren Seilproben. Als weiterer Grund wird die umfassende Bewitterung der gesamten Probenoberfläche gesehen, während an statischen Proben nur die Bewitterung eines Umfangsausschnittes an einem Seilprobensegment erfolgt. Da Faserseile bei der Anwendung im Outdoorbereich zumeist einer Bewegung unterliegen, ist die Bewitterung umlaufender Seilproben eine bessere Annäherung an reale Nutzungsbedingungen, als eine Bewitterung starrer Seilproben. Dies konnte durch die Zugversuche hinreichend belegt werden. Somit konnte auch gezeigt werden, dass das im Projekt entwickelte Verfahren zur Bewitterung umlaufender Seilproben für die Beurteilung der Ablegereife von Faserseilen besser geeignet ist, als eine Bewitterung nach dem bisherigen Stand der Technik. Es konnte ebenfalls gezeigt werden, das es gelang, die technischen Parameter für die künstliche Bewitterung umlaufender Seilproben so zu quantifizieren, dass diese an den Proben annähernd die gleiche Minderung der ertragbaren Zugkräfte hergerufen hat, wie die natürliche Bewitterung umlaufender Seilproben. Die Gültigkeitsüberprüfung dieser Parameterwerte für andere Materialpaarungen wie beispielsweise Vektran ${ }^{\circledR}$-Kern/PES-Mantel ist Gegenstand weiterführender Entwicklungen.

Im Dezember 2015 erfolgte die Einreichung eines Patentes „Vorrichtung und Verfahren zur Prüfung der Bewitterung von Faserseilen sowie der Beurteilung der Ablegereife“. Dies stellt einerseits die erste Veröffentlichung der Projektergebnisse dar, zum anderen sollen somit entsprechende Schutzrechte gesichert werden. Das Patent wurde erteilt. 